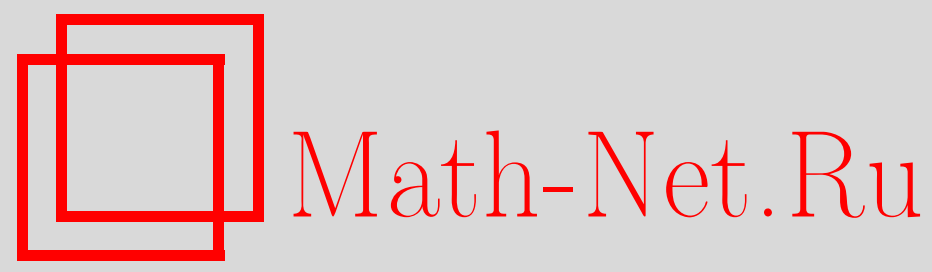

А. О. Логачев, Об одном рекурсивном классе платовидных булевых функций, Дискрет. матем., 2010, том 22, выпуск 4, 20-33

DOI: https://doi.org/10.4213/dm1116

Использование Общероссийского математического портала Math-Net.Ru подразумевает, что вы прочитали и согласны с пользовательским соглашением http://www . mathnet.ru/rus/agreement

Параметры загрузки:

IP: 54.198 .64 .247

26 апреля 2023 г., $15: 34: 38$ 


\title{
Об одном рекурсивном классе платовидных булевых функций
}

\author{
() 2010 г. А. О. Логачев
}

\begin{abstract}
В данной работе исследуются свойства класса платовидных булевых функций, носитель спектра которых задается рекурсивным классом матриц. Для этой последовательности носителей спектра получено точное число функций с таким носителем спектра. Показано также, что множество этих функций являются классом эквивалентности относительно группы сдвигов функций с таким носителем спектра.
\end{abstract}

\section{1. Введение}

Платовидные функции представляют большой интерес с точки зрения исследования свойств таких классов функций, как максимально-нелинейные, корреляционно-иммунные, устойчивые и другие.

Важной характеристикой платовидных булевых функций является множество векторов, соответствующих ненулевым коэффициентам Уолша этой функции. Это множество называется носителем спектра. Количественными характеристиками носителя спектра являются его мощность, ранг и аффинный ранг.

Широко известный и важный класс бент-функций можно рассматривать как частный случай платовидных, когда носитель спектра совпадает со всем множеством двоичных наборов. Бент-функции существуют тогда и только тогда, когда число переменных четно. В настоящее время точное число бент-функций от $n$ переменных известно только при $n=2,4,6,8$.

В данной работе изучаются свойства платовидных булевых функций специфического рекурсивного класса и их связь с рекурсивным классом матриц, строки которых задают носитель спектра платовидной функции.

Для этой последовательности носителей спектра подсчитано точное число функций с таким носителем спектра и показано, что множество таких функций совпадает с множеством функций, получаемых сдвигами из функции, задающей рекурсивную последовательность. Таким образом, по-видимому, впервые для бесконечной последовательности носителей спектра растущей мощности получено точное растущее число функций с таким носителем спектра. 


\section{2. Основные определения}

Мы рассматриваем векторное пространство $F_{2}^{n}$ наборов длины $n$ с компонентами из конечного поля $F_{2}$ из двух элементов 0 и 1 , операции сложения и умножения в котором вводятся как обычные операции сложения и умножения чисел 0 и 1 по модулю 2. Булева функция от $n$ переменных - это отображение из $F_{2}^{n}$ в $F_{2}$. В дальнейшем мы будем обозначать набор из $F_{2}^{n}$ буквой без нижнего индекса, а компоненту этого набора - той же буквой с нижним индексом, указывающим на порядковый номер этой компоненты в наборе. Наборы $x^{\prime}$ и $x^{\prime \prime}$ называются соседними, если они различаются только в $i$-й компоненте, $i=1, \ldots, n$. Переменная $x_{i}$ называется фиктивной для функции $f$, если для любых наборов $x^{\prime}$ и $x^{\prime \prime}$, соседних по $i$-й компоненте, выполнено равенство $f\left(x^{\prime}\right)=f\left(x^{\prime \prime}\right)$. Расстоянием Хэмминга $d\left(x^{\prime}, x^{\prime \prime}\right)$ между двумя наборами $x^{\prime}$ и $x^{\prime \prime}$ называется число компонент, в которых наборы $x^{\prime}$ и $x^{\prime \prime}$ различаются. Для заданной функции $f$ из $F_{2}^{n}$ минимум расстояний $d(f, l)$, где $l$ пробегает множество всех аффинных функций из $F_{2}^{n}$, называется нелинейностью функции $f$ и обозначается через $\mathrm{nl}(f)$. Подфункцией булевой функции $f$ называется функция $f^{\prime}$, полученная подстановкой в $f$ некоторых констант 0 и 1 вместо некоторых переменных.

Хорошо известно, что функция $f$, заданная на $F_{2}^{n}$, имеет единственное полиномиальное представление над $F_{2}$, степень которого по каждой переменной не превосходит 1 , а именно,

$$
f\left(x_{1}, \ldots, x_{n}\right)=\bigotimes_{\left(a_{1}, \ldots, a_{n}\right) \in F_{2}^{n}} g\left(a_{1}, \ldots, a_{n}\right) x_{1}^{a_{1}} \ldots x_{n}^{a_{n}},
$$

где $g$ - это тоже функция над $F_{2}^{n}$. Такое полиномиальное представление $f$ называется алгебраической нормальной формой (сокращенно АНФ) функции, а каждое выражение $x_{1}^{a_{1}} \ldots x_{n}^{a_{n}}$ называется слагаемым в АНФ функции $f$ (в русскоязычной литературе АНФ также называют полиномом Жегалкина). Отображение $f(x) \rightarrow g(x)$ иногда называют преобразованием Мебиуса.

Алгебраическая степень функции $f$, обозначаемая через $\operatorname{deg}(f)$, определяется как число переменных в самом длинном слагаемом в АНФ функции $f$.

$\operatorname{Bec} \operatorname{wt}(f)$ функции $f$ над $F_{2}^{n}$ - это число наборов $x$ из $F_{2}^{n}$, для которых $f(x)=1$. Функция $f$ называется уравновешенной, если

$$
\operatorname{wt}(f)=\operatorname{wt}(f \oplus 1)=2^{n-1},
$$

то есть если функция принимает значения 0 и 1 на одинаковом числе наборов.

Пусть $x=\left(x_{1}, \ldots, x_{n}\right)$ и $u=\left(u_{1}, \ldots, u_{n}\right)$ - это наборы длины $n$ на $F_{2}^{n}$. Скалярное произведение $x$ и $u$ - это функция $\langle x, u\rangle$, которая определяется равенством

$$
\langle x, u\rangle=\sum_{i=1}^{n} x_{i} u_{i}
$$

где сложение и умножение берутся над $F_{2}$. Под суммой $x+u$ двух двоичных наборов $x$ и $u$ мы понимаем набор, получаемый их покомпонентным суммированием над $F_{2}$.

Преобразованием Уолша булевой функции $f$ называется целочисленная функция над $F_{2}^{n}$, определяемая следующим образом:

$$
W_{f}(u)=\sum_{x \in F_{2}^{n}}(-1)^{f(x)+\langle u, x\rangle} .
$$


Для каждого $u \in F_{2}^{n}$ значение $W_{f}(u)$ называется коэффициентом Уолша. Коэффициенты Уолша будем называть спектральными коэффициентами, а совокупность всех $2^{n}$ коэффициентов Уолша - спектром булевой функции. Множество $S_{f}$ всех наборов $u$ таких, что $W_{f}(u) \neq 0$, называется носителем спектра функции $f$. Матрицей носителя спектра булевой функции называется матрица, строками которой по одному разу являются все наборы из носителя спектра. Коэффициенты Уолша удовлетворяют формуле обращения

$$
(-1)^{f(x)}=2^{-n} \sum_{u \in F_{2}^{n}} W_{f}(u)(-1)^{\langle u, x\rangle}
$$

и равенству Парсеваля

$$
\sum_{u \in F_{2}^{n}} W_{f}^{2}(u)=2^{2 n}
$$

Нелинейность булевой функции $f$ выражается через ее коэффициенты Уолша следующим образом:

$$
\mathrm{nl}(f)=2^{n-1}-\frac{1}{2} \max _{u \in F_{2}^{n}}\left|W_{f}(u)\right| .
$$

Булева функция называется бент-функцией, если значение коэффициентов Уолша на всех наборах равно $\pm 2^{n / 2}$. Бент-функции существуют при всех четных $n$, а при нечетных не существуют. Бент-функции являются функциями с максимально возможной нелинейностью $\left.2^{n-1}-2^{n /(2-1}\right)$ среди всех функций от $n$ переменных при четном $n$. Булева функция называется платовидной, если ее коэффициенты Уолша принимают ровно три возможных значения: 0 и $\pm 2^{c}$ для некоторого $c$. Платовидные функции представляют большой интерес для изучения бент-функций (например, потому, что при разложении бент-функции по переменной возникают две платовидные функции), а также потому, что многие криптографически важные функции являются платовидными (например, $m$-устойчивые функции с максимально возможной для них нелинейностью $2^{n-1}-2^{m+1}$ ). Для платовидных функций положим

$$
\varphi(x)=2^{-c} W_{f}(x) .
$$

Тогда для любого $x \in F_{2}^{n}$ величина $\varphi(x)$ может принимать только три значения: $0,-1$, 1. Множество $S_{f}$ всех наборов $u$ таких, что $W_{f}(u) \neq 0$, называется носителем спектра платовидной функции. Множество всех наборов, на которых $\varphi(x)=-1$, будем обозначать через $T^{-}$, а множество всех наборов, на которых $\varphi(x)=1$, будем обозначать через $T^{+}$. Из равенства Парсеваля сразу следует, что мощность носителя спектра равна $4^{n-c}$.

В настоящей работе одним из основных инструментов исследования является следующая теорема Титсворта.

Теорема 1. Набор чисел $W_{f}(u)$, заданный на $F_{2}^{n}$, является набором коэффициентов Уолша булевой функиии $f$ тогда и только тогда, когда для любого ненулевого $s \in F_{2}^{n}$

$$
\sum_{u \in F_{2}^{n}} W_{f}(u) W_{f}(u+s)=0
$$

и, кроме того, выполнено равенство Парсеваля

$$
\sum_{u \in F_{2}^{n}} W_{f}^{2}(u)=2^{2 n}
$$




\section{3. Описание рекурсивного класса булевых функций}

Рассмотрим рекурсивный класс булевых функций, введенный в [3]:

(1) $n_{k+1}=2 n_{k}+2, \quad k=0,1, \ldots, \quad n_{0}=4$;

(2) $f_{0} \in P_{2}^{(4)}$ и имеет вид

$$
f_{0}\left(x_{1}, \ldots, x_{4}\right)=\left(x_{1}+x_{2}\right)\left(x_{3}+x_{4}\right)+x_{1}+x_{3} ;
$$

(3) $f_{k+1} \in P_{2}^{\left(n_{k+1}\right)}, n_{k+1}=2 n_{k}+2$ и выражается через $f_{k}$ следующим образом:

$$
\begin{aligned}
(x, y, z) & \in F_{2}^{n_{k+1}}, \quad x, y \in F_{2}^{n_{k}}, \quad z \in F_{2}^{2}, \\
f_{k+1}(x, y, z)= & \left(z_{1}+z_{2}+1\right)\left(f_{k}(x)+\left\langle 1^{n_{k}}, y\right\rangle\right) \\
& \quad+\left(z_{1}+z_{2}\right)\left(f_{k}(y)+\left\langle 1^{n_{k}}, x\right\rangle\right)+z_{1} .
\end{aligned}
$$

В дальнейшем через $A_{k}$ будем обозначать матрицу носителя спектра для функции $f_{k}$. Выразим $n_{k}$ через $k$ :

$$
\begin{aligned}
n_{k} & =2(\ldots(2(2 \cdot 4+2)+2) \ldots)+2 \\
& =2^{k} \cdot 4+2^{k}+2^{k-1}+\ldots+2 \\
& =5 \cdot 2^{k}+2^{k-1}+2^{k-2}+\ldots+2 \\
& =6 \cdot 2^{k}-2 .
\end{aligned}
$$

Посмотрим, как будут выглядеть коэффициенты Уолша $W_{f}$ для такого класса булевых функций.

Пусть $(u, v, w) \in F_{2}^{n_{k}} \times F_{2}^{n_{k}} \times F_{2}^{2}$, тогда

$$
\begin{aligned}
W_{f_{k+1}}(u, v, w)= & \sum_{x, y, z}(-1)^{f_{k+1}(x, y, z)+\langle(u, v, w),(x, y, z)\rangle} \\
= & \sum_{z} \sum_{y} \sum_{x}(-1)^{f_{k+1}(x, y, z)+\langle(u, v, w),(x, y, z)\rangle} \\
= & \sum_{y} \sum_{x}(-1)^{f_{k}(x)+\left\langle 1^{n_{k}}, y\right\rangle+\langle u, x\rangle+\langle v, y\rangle} \\
& \quad+\sum_{y} \sum_{x}(-1)^{f_{k}(y)+\left\langle 1^{n_{k}}, x\right\rangle+1+\langle u, x\rangle+\langle v, y\rangle+w_{1}} \\
& +\sum_{y} \sum_{x}(-1)^{f_{k}(y)+\left\langle 1^{n_{k}}, x\right\rangle+\langle u, x\rangle+\langle v, y\rangle+w_{2}} \\
& \quad+\sum_{y} \sum_{x}(-1)^{f_{k}(x)+\left\langle 1^{n_{k}}, y\right\rangle+1+\langle u, x\rangle+\langle v, y\rangle+w_{1}+w_{2} .}
\end{aligned}
$$

Рассмотрим теперь каждое слагаемое:

$$
\begin{aligned}
\sum_{y} \sum_{x}(-1)^{f_{k}(x)+\left\langle 1^{n_{k}}, y\right\rangle+\langle u, x\rangle+\langle v, y\rangle} & \\
& =\sum_{y}(-1)^{\left\langle 1^{n_{k}}+v, y\right\rangle} \sum_{x}(-1)^{f_{k}(x)+\langle u, x\rangle} \\
& =W_{f_{k}}(u) \sum_{y}(-1)^{\left\langle 1^{n_{k}}+v, y\right\rangle} ;
\end{aligned}
$$




$$
\begin{aligned}
& \sum_{y} \sum_{x}(-1)^{f_{k}(y)+\left\langle 1^{n_{k}}, x\right\rangle+1+\langle u, x\rangle+\langle v, y\rangle+w_{1}}=(-1)^{w_{1}+1} \sum_{y}(-1)^{f_{k}(y)+\langle v, y\rangle} \sum_{x}(-1)^{\left\langle 1^{n_{k}}+u, x\right\rangle} \\
&=(-1)^{w_{1}+1} W_{f_{k}}(v) \sum_{x}(-1)^{\left\langle 1^{m} n_{k}+u, x\right\rangle} ; \\
& \sum_{y} \sum_{x}(-1)^{f_{k}(y)+\left\langle 1^{n_{k}}, x\right\rangle+\langle u, x\rangle+\langle v, y\rangle+w_{2}} \\
&=(-1)^{w_{2}} \sum_{y}(-1)^{f_{k}(y)+\langle v, y\rangle} \sum_{x}(-1)^{\left\langle 1^{n_{k}}+u, x\right\rangle} \\
&=(-1)^{w_{2}} W_{f_{k}}(v) \sum_{x}(-1)^{\left\langle 1^{n_{k}}+u, x\right\rangle} ; \\
& \sum_{y} \sum_{x}(-1)^{f_{k}(x)+\left\langle 1^{n_{k}}, y\right\rangle+1+\langle u, x\rangle+\langle v, y\rangle+w_{1}+w_{2}} \\
&=(-1)^{w_{1}+w_{2}+1} \sum_{x}(-1)^{f_{k}(x)+\langle u, x\rangle} \sum_{y}(-1)^{\left\langle 1^{n_{k}}+v, y\right\rangle} \\
&=(-1)^{w_{1}+w_{2}+1} W_{f_{k}}(u) \sum_{y}(-1)^{\left\langle 1^{n_{k}}+v, y\right\rangle} .
\end{aligned}
$$

В итоге получаем выражение коэффициентов Уолша функции $f_{k+1}$ через коэффициенты Уолша функции $f_{k}$ :

$$
\begin{aligned}
W_{f_{k+1}}(u, v, w)= & W_{f_{k}}(u) \sum_{y}(-1)^{\left\langle 1^{n} k+v, y\right\rangle} \\
& +(-1)^{w_{1}+1} W_{f_{k}}(v) \sum_{x}(-1)^{\left\langle 1^{n_{k}}+u, x\right\rangle} \\
& +(-1)^{w_{2}} W_{f_{k}}(v) \sum_{x}(-1)^{\left\langle 1^{n_{k}}+u, x\right\rangle} \\
& +(-1)^{w_{1}+w_{2}+1} W_{f_{k}}(u) \sum_{y}(-1)^{\left\langle 1^{n_{k}}+v, y\right\rangle} .
\end{aligned}
$$

Лемма 1. Пусть $f_{k}$ из определенного выше рекурсивного класса, тогда

$$
W_{f_{k}}(u, v, w) \in\left\{0, \pm 2^{n_{k}-k-1}\right\}, \quad W_{f_{k}}\left(1^{n_{k}}\right)=0 .
$$

Доказательство. Функция

$$
f_{0}\left(x_{1}, x_{2}, x_{3}, x_{4}\right)=\left(x_{1}+x_{2}\right)\left(x_{3}+x_{4}\right)+x_{1}+x_{3}
$$

есть платовидная булева функция, коэффициенты Уолша которой равны $\left\{0, \pm 2^{3}\right\}$, и матрица носителя спектра имеет вид

$$
A_{0}=\left(\begin{array}{llll}
1 & 0 & 1 & 0 \\
1 & 0 & 0 & 1 \\
0 & 1 & 1 & 0 \\
0 & 1 & 0 & 1
\end{array}\right)
$$

Это будет служить основанием индукции при $k=0$. Считаем, что для $k$ предположение выполнено, докажем его для $k+1$. 
Перепишем полученную формулу для $W_{f_{k+1}}$ в следующем виде:

$$
\begin{aligned}
W_{f_{k+1}}(u, v, w)=W_{f_{k}}(u)\left(\sum_{y}(-1)^{\left\langle 1^{n} k+v, y\right\rangle}\right)\left(1+(-1)^{w_{1}+w_{2}+1}\right) & \\
& +W_{f_{k}}(v)\left(\sum_{x}(-1)^{\left\langle 1^{n_{k}}+u, x\right\rangle}\right)\left((-1)^{w_{1}+1}+(-1)^{w_{2}}\right) .
\end{aligned}
$$

Рассмотрим $(u, v, w) \in F_{2}^{n_{k}} \times F_{2}^{n_{k}} \times F_{2}^{2}$. Если $u \neq 1^{n_{k}}, v \neq 1^{n_{k}}$, то

$$
W_{f_{k+1}}(u, v, w)=0 .
$$

Если $v \neq 1^{n_{k}}, w_{1}=w_{2}$, то

$$
W_{f_{k+1}}\left(1^{n_{k}}, v, w\right)=0 .
$$

Если $v \neq 1^{n_{k}}, w_{1} \neq w_{2}$, то

$$
W_{f_{k+1}}\left(1^{n_{k}}, v, w\right)=W_{f_{k}}(v)(-1)^{w_{2}} 2^{n_{k}+1} .
$$

Если $u \neq 1^{n_{k}}, w_{1}=w_{2}$, то

$$
W_{f_{k+1}}\left(u, 1^{n_{k}}, w\right)=0 .
$$

Если $u \neq 1^{n_{k}}, w_{1} \neq w_{2}$, то

$$
W_{f_{k+1}}\left(u, 1^{n_{k}}, w\right)=W_{f_{k}}(u) 2^{n_{k}+1} .
$$

Из того, что по предположению индукции $W_{f_{k}}\left(1^{n_{k}}\right)=0$, следует справедливость равенства

$$
W_{f_{k+1}}\left(1^{n_{k}}, 1^{n_{k}}, w\right)=0
$$

и это одновременно доказывает, что

$$
W_{f_{k+1}}\left(1^{n_{k+1}}\right)=0 \text {. }
$$

Разобрав все случаи, получаем, что либо

$$
W_{f_{k+1}}(u, v, w)=0,
$$

либо

$$
W_{f_{k+1}}(u, v, w)= \pm 2^{n_{k}+1} 2^{n_{k}-k-1}= \pm 2^{n_{k+1}-(k+1)-1} .
$$

Лемма доказана.

Таким образом, все функции из введенного выше рекурсивного класса суть платовидные булевы функции, для функции $f_{k}$ из этого класса коэффициенты Уолша равны 
$\left\{0, \pm 2^{n_{k}-k-1}\right\}$, а матрица носителя спектра имеет размер $\left(4^{k+1} \times\left(6 \cdot 2^{k}-2\right)\right)$ и выглядит следующим образом:

$$
A_{k}=\left(\begin{array}{cccc} 
& & 0 & 1 \\
A_{k-1} & 1 & \vdots & \vdots \\
& & 0 & 1 \\
& & 1 & 0 \\
A_{k-1} & 1 & \vdots & \vdots \\
& & 1 & 0 \\
& & 0 & 1 \\
1 & A_{k-1} & \vdots & \vdots \\
& & 0 & 1 \\
& & 1 & 0 \\
1 & A_{k-1} & \vdots & \vdots \\
& & 1 & 0
\end{array}\right),
$$

где $A_{k-1}$ есть матрица носителя спектра для функции $f_{k-1}$.

\section{4. Число функций с носителем спектра, задаваемым матрицей $A_{k}$}

В предыдущей главе мы рассматривали матрицу $A_{k}$, являющуюся матрицей носителя спектра функции $f_{k}$. Однако функция $f_{k}$, возможно, является не единственной функцией с носителем спектра, задаваемым матрицей $A_{k}$. Обозначим множество всех таких функций через $M_{k}$. В этом разделе мы исследуем вопрос о числе $\left|M_{k}\right|$ таких функций.

По носителю спектра платовидной функции модули коэффициентов Уолша на всех наборах восстанавливаются однозначно. Поэтому для задания функции достаточно определить знаки коэффициентов Уолша на всех наборах из носителя спектра, то есть достаточно сопоставить каждой строке матрицы $A_{k}$ знак + или - . Для этого воспользуемся теоремой Титсворта.

Рассмотрим матрицу $A_{k}$, каждой строке $u$ этой матрицы присвоим некоторое значение $W_{f}(u)$. Так как матрица имеет размеры $4^{k+1} \times\left(6 \cdot 2^{k}-2\right)$, из равенства Парсеваля (2) находим абсолютное значение коэффициентов Уолша на всех наборах из носителя спектра:

$$
\begin{aligned}
\sum_{u \in F_{2}^{n}} W_{f}^{2}(u)=2^{2 n_{k}} & \Longleftrightarrow 4^{k+1} W_{f}^{2}(u)=2^{2\left(6 \cdot 2^{k}-2\right)} \\
& \Longleftrightarrow\left|W_{f}(u)\right|=2^{6 \cdot 2^{k}-k-3} .
\end{aligned}
$$

Далее нам надо, чтобы $W_{f}(u)$ удовлетворяли равенству (1). Воспользуемся функцией $\varphi(x)$, тогда это равенство упрощается и принимает вид

$$
\sum_{u \in F_{2}^{n}} \varphi(u) \varphi(u+s)=0, \quad s \neq 0 .
$$

Таким образом, мы пришли к тому, что найдя число сопоставлений всеми возможными способами + и - строкам матрицы $A_{k}$ так, чтобы выполнялось соотношение (3), мы 
получим число платовидных функций, для которых эти матрицы являются матрицами носителя спектра.

Для заданного $s \in F_{2}^{n}, s \neq 0$, будем называть совокупность всех пар наборов $(u, u+s)$ таких, что $W_{f}(u) W_{f}(u+s) \neq 0$, пучком параллельных отрезков вдоль направления $s$ и обозначать каждый из них $u-(u+s)$ или $n(u)-n(u+s)$, где $n(u)-$ номер строки матрицы $A_{k}$, соответствующий набору $u$.

Рассмотрим матрицу

$$
A_{0}=\left(\begin{array}{llll}
1 & 0 & 1 & 0 \\
1 & 0 & 0 & 1 \\
0 & 1 & 1 & 0 \\
0 & 1 & 0 & 1
\end{array}\right)
$$

для нее соотношение (3) принимает вид

$$
\varphi(u) \varphi(u+s)+\varphi(v) \varphi(v+s)=0, \quad s \neq 0 .
$$

Пучков параллельных отрезков в этом случае будет три: $\{(1,2),(3,4)\} ;\{(1,3),(2,4)\}$; $\{(1,4),(2,3)\}$.

Очевидно, что равенство выполняется тогда и только тогда, когда одному набору мы сопоставляем -, а трем другим + , и наоборот, одному + , трем другим -. Всего таких возможностей 8. В итоге получаем, что матрице $A_{0}$, как матрице носителя спектра, соответствует $\left|M_{0}\right|=8$ различных платовидных булевых функций.

Рассмотрим опять матрицу $A_{k}$. Для нее введем понятие полосы. Напомним, что эта матрица имеет $4^{k+1}$ строк. Первой полосой будем называть первую четверть строк матрицы $A_{k}$. Второй, третьей и четвертой полосой будем называть соответственно вторую, третью и четвертую четверти строк матрицы $A_{k}$. Для краткости, вместо первая полоса будем писать I и аналогично для остальных полос.

Пусть $\alpha \in I$ и $\beta \in$ III, тогда направлению $s=\alpha+\beta$ будет соответствовать пучок $\alpha-\beta$, состоящий, как легко видеть из структуры матрицы, только из двух пар наборов $(\alpha, \beta)$ и $\left(\alpha^{*}, \beta^{*}\right)$, где $\alpha^{*} \in \mathrm{II}$ и $\alpha+\alpha^{*}=(0, \ldots, 0,1,1)$, а $\beta^{*} \in \mathrm{IV}$ и $\beta+\beta^{*}=(0, \ldots, 0,1,1)$.

Таким образом, соотношение (3) принимает вид

$$
\varphi(\alpha) \varphi(\beta)+\varphi\left(\alpha^{*}\right) \varphi\left(\beta^{*}\right)=0 \text {. }
$$

Пусть $\varphi(\alpha)$ и $\varphi\left(\alpha^{*}\right)$ одного знака, тогда для того, чтобы соотношение (3) было выполнено, $\varphi(\beta)$ и $\varphi\left(\beta^{*}\right)$ должны быть разных знаков. Зафиксировав $\varphi(\alpha)$ и $\varphi\left(\alpha^{*}\right)$ одного знака и пробегая набором $\beta$ все строки третьей полосы, мы получим, что знаки на соответствующих наборах полос III и IV должны быть противоположными. Теперь возьмем и зафиксируем $\varphi(\beta)$ и $\varphi\left(\beta^{*}\right)$, пробежав набором $\alpha$ все строки первой полосы, получим, что знаки на соответствующих наборах полос I и II должны быть одинаковыми.

Пусть $\varphi(\alpha)$ и $\varphi\left(\alpha^{*}\right)$ разных знаков, тогда для того, чтобы соотношение (3) было выполнено, $\varphi(\beta)$ и $\varphi\left(\beta^{*}\right)$ должны быть одного знака. Зафиксировав $\varphi(\alpha)$ и $\varphi\left(\alpha^{*}\right)$ разных знаков и пробегая набором $\beta$ все строки третьей полосы, мы получим, что знаки на соответствующих наборах полос III и IV должны быть одинаковыми. Теперь возьмем и зафиксируем $\varphi(\beta)$ и $\varphi\left(\beta^{*}\right)$. Тогда, пробежав набором $\alpha$ все строки первой полосы, получим, что знаки на соответствующих наборах полос I и II должны быть разными.

Пусть $\alpha \in \mathrm{I}$ и $\beta \in \mathrm{IV}$, тогда направлению $s=\alpha+\beta$ будет соответствовать пучок $\alpha-\beta$, состоящий, как легко видеть из структуры матрицы, только из двух пар наборов $(\alpha, \beta)$ и $\left(\alpha^{*}, \beta^{*}\right)$, где $\alpha^{*} \in$ II и $\alpha+\alpha^{*}=(0, \ldots, 0,1,1)$, а $\beta^{*} \in$ III и $\beta+\beta^{*}=(0, \ldots, 0,1,1)$. 
Таким образом, соотношение (3) принимает вид

$$
\varphi(\alpha) \varphi(\beta)+\varphi\left(\alpha^{*}\right) \varphi\left(\beta^{*}\right)=0 .
$$

Пусть $\varphi(\alpha)$ и $\varphi\left(\alpha^{*}\right)$ одного знака, тогда для того, чтобы соотношение (3) было выполнено, $\varphi(\beta)$ и $\varphi\left(\beta^{*}\right)$ должны быть разных знаков. Зафиксировав $\varphi(\alpha)$ и $\varphi\left(\alpha^{*}\right)$ одного знака и пробегая набором $\beta$ все строки четвертой полосы, мы получим, что знаки на соответствующих наборах полос III и IV должны быть противоположными. Теперь возьмем и зафиксируем $\varphi(\beta)$ и $\varphi\left(\beta^{*}\right)$. Тогда, пробежав набором $\alpha$ все строки первой полосы, получим, что знаки на соответствующих наборах полос I и II должны быть одинаковыми.

Пусть $\varphi(\alpha)$ и $\varphi\left(\alpha^{*}\right)$ разных знаков, тогда для того, чтобы соотношение (3) было выполнено, $\varphi(\beta)$ и $\varphi\left(\beta^{*}\right)$ должны быть одного знака. Зафиксировав $\varphi(\alpha)$ и $\varphi\left(\alpha^{*}\right)$ разных знаков и пробегая набором $\beta$ все строки четвертой полосы, мы получим, что знаки на соответствующих наборах полос III и IV должны быть одинаковыми. Теперь возьмем и зафиксируем $\varphi(\beta)$ и $\varphi\left(\beta^{*}\right)$. Тогда, пробежав набором $\alpha$ все строки первой полосы, получим, что знаки на соответствующих наборах полос I и II должны быть разными.

Таким образом, получаем два случая. В первом случае расстановки на первой и второй полосах совпадают, при этом на третьей и четвертой они противоположны. Во втором случае расстановки на первой и второй полосах противоположны, при этом на третьей и четвертой полосах расстановки совпадают.

Мы рассмотрели все пучки $\alpha-\beta$ такие, что $\alpha \in \mathrm{I}$, II, а $\beta \in \mathrm{III}$, IV. Пусть $\alpha \in \mathrm{I}$ и $\beta \in \mathrm{I}$, тогда направлению $s=\alpha+\beta$ будет соответствовать пучок $\alpha-\beta$, состоящий, как легко видеть из структуры матрицы, из двух множеств пар $(\alpha, \beta)$ и $\left(\alpha^{*}, \beta^{*}\right)$, где $\alpha^{*} \in$ II и $\alpha+\alpha^{*}=(0, \ldots, 0,1,1)$, а $\beta^{*} \in$ II и $\beta+\beta^{*}=(0, \ldots, 0,1,1)$. Из этого следует, что соотношение (3) принимает вид

$$
\sum_{\alpha \in \mathrm{I}} \varphi(\alpha) \varphi(\alpha+s)+\sum_{\alpha^{*} \in \mathrm{II}} \varphi\left(\alpha^{*}\right) \varphi\left(\alpha^{*}+s\right)=0, \quad \forall s=\alpha+\beta .
$$

В предыдущем рассуждении мы выяснили, что расстановки плюсов и минусов на первой и второй полосах должны совпадать или быть противоположными. Когда расстановки на полосах I и II совпадают, обе суммы в выражении (5) совпадают. Точно так же они совпадают и тогда, когда расстановки на I и II полосах противоположны. Поэтому

$$
2 \sum_{\alpha \in \mathrm{I}} \varphi(\alpha) \varphi(\alpha+s)=0, \quad \forall s=\alpha+\beta
$$

В этом выражении участвуют наборы только из первой полосы. Очевидно, что оно выполнено тогда и только тогда, когда расстановки на первой полосе совпадают с расстановками на матрице $A_{k-1}$.

Пусть $\alpha \in \mathrm{I}$ и $\beta \in \mathrm{II}$, где $\alpha+\beta=(0, \ldots, 0,1,1)$. Направлению $s=\alpha+\beta$ будет соответствовать пучок $\alpha-\beta$, состоящий, как легко видеть из структуры матрицы, из двух множеств пар $(\alpha, \beta)$ и $\left(\alpha^{*}, \beta^{*}\right)$, где $\alpha^{*} \in \mathrm{III}, \beta^{*} \in \mathrm{IV}$ и $\alpha^{*}+\beta^{*}=(0, \ldots, 0,1,1)$. В каждой половине все наборы распределены по парам, из предыдущих рассуждений следует, что в одной из половин все пары одного знака, а в другой противоположного. Поэтому для каждого слагаемого в соотношении (3) будет и ему противоположное слагаемое, так что (3) будет выполнено.

Пусть $\alpha \in \mathrm{I}$ и $\beta \in \mathrm{II}$, где $\alpha+\beta \neq(0, \ldots, 0,1,1)$. Направлению $s=\alpha+\beta$ будет соответствовать пучок $\alpha-\beta$. Этот пучок, как легко видеть из структуры матрицы, состоит 
из двух множеств пар $(\alpha, \beta)$ и $\left(\alpha^{*}, \beta^{*}\right)$, где

$$
\begin{aligned}
\alpha+\alpha^{*} & =(0, \ldots, 0,1,1), \\
\beta+\beta^{*} & =(0, \ldots, 0,1,1) .
\end{aligned}
$$

Соотношение (3) принимает вид

$$
\sum_{\alpha \in \mathrm{I}} \varphi(\alpha) \varphi(\alpha+s)+\sum_{\alpha^{*} \in \mathrm{II}} \varphi\left(\alpha^{*}\right) \varphi\left(\alpha^{*}+s\right)=0, \quad \forall s=\alpha+\beta
$$

Ранее мы выяснили, что расстановки плюсов и минусов на первой и второй полосах должны совпадать или быть противоположными. В обоих случаях суммы равны, и выражение (7) можно переписать в виде

$$
2 \sum_{\alpha \in \mathrm{I}} \varphi(\alpha) \varphi\left(\alpha^{*}+s\right)=0, \quad \forall s=\alpha+\beta
$$

В этом выражении участвуют наборы только из первой полосы. Получаем, что оно выполнено тогда и только тогда, когда расстановки на первой полосе совпадают с расстановками в матрице $A_{k-1}$.

Случаи, когда $\alpha \in \mathrm{II}$ и $\beta \in \mathrm{II} ; \alpha \in \mathrm{III}$ и $\beta \in \mathrm{III} ; \alpha \in \mathrm{III}$ и $\beta \in \mathrm{IV} ; \alpha \in \mathrm{IV}$ и $\beta \in \mathrm{IV}$, либо включены в уже разобранные случаи, либо являются их точным повторением. Таким образом, были разобраны все возможные направления в матрице $A_{k}$, выведено правило расстановки + и - в матрице $A_{k}$ : на двух полосах одной половины матрицы расстановки совпадают, а расстановки на двух полосах из другой половины различаются, причем расстановки на любой полосе матрицы $A_{k}$ должны совпадать с расстановками на матрице $A_{k-1}$. Таким образом, доказана следующая лемма.

Лемма 2. Для числа функичий $\left|M_{k}\right|$ с матрицей носителя спектра $A_{k}$ имеет место рекурсивное соотношение

$$
\left|M_{k+1}\right|=2\left|M_{k}\right|^{2}
$$

Теорема 2. Для числа функичй $\left|M_{k}\right|$ с матрицей носителя спектра $A_{k}$ имеет место формула

$$
\left|M_{k}\right|=2^{4 \cdot 2^{k}-1} .
$$

Доказательство. Ранее было показано, что $\left|M_{0}\right|=8$. Воспользовавшись рекурсивным выражением для $\left|M_{k}\right|$ из леммы 2, получим, что

$$
\begin{aligned}
\left|M_{k+1}\right| & =2\left|M_{k}\right|^{2}=2\left(2\left|M_{k-1}\right|^{2}\right)^{2}=2 \cdot 2^{2} t\left|M_{k-1}\right|^{4} \\
& =2 \cdot 2^{2}\left(2\left|M_{k-2}\right|^{2}\right)^{4}=2 \cdot 2^{2} 2^{4}\left|M_{k-2}\right|^{8}=\ldots \\
& =2 \cdot 2^{2} 2^{4} \ldots 2^{2^{k}}\left|M_{0}\right|^{2^{k+1}} \\
& =2^{1+2+4+\ldots+2^{k}}\left(2^{3}\right)^{2^{k+1}} \\
& =2^{2^{k+1}-1} 2^{3 \cdot 2^{k+1}}=2^{2^{k+3}-1},
\end{aligned}
$$

то есть

$$
\left|M_{k}\right|=2^{4 \cdot 2^{k}-1} .
$$

Теорема доказана. 


\section{5. Сдвиги функций из рекурсивного класса}

Вернемся к рекурсивному классу функций. Будем действовать группой $T_{n}$ на функции следующим образом: $g_{t} \in T_{n}, g_{t}: f(x) \rightarrow f(x+t),\left|T_{n}\right|=2^{n}$. Пусть

$$
J_{T_{n}}(f)=\left\{t \in F_{2}^{n}: f(x)+f(x+t)=0\right\}
$$

есть группа инерции функции $f$ относительно группы $T_{n}$,

$$
L_{f}=\left\{a \in F_{2}^{n}: f(x)+f(x+a)=c\right\},
$$

где $c$ - константа, есть группа линейности функции $f$.

Лемма 3. Введенное выше действие группь $T_{n}$ не меняет носитель спектра булевой функции.

Доказательство. Рассмотрим $f(x)$ и введем обозначение

$$
f(x+u)=f^{\prime}(x) .
$$

Справедливы равенства

$$
\begin{aligned}
W_{f^{\prime}}(v) & =\sum_{x \in F_{2}^{n}}(-1)^{f^{\prime}(x)+\langle v, x\rangle} \\
& =\sum_{x \in F_{2}^{n}}(-1)^{f(x+u)+\langle v, x\rangle} \\
& =\sum_{z \in F_{2}^{n}}(-1)^{f(z)+\langle v, z+u\rangle} \\
& =(-1)^{\langle v, u\rangle} \sum_{z \in F_{2}^{n}}(-1)^{f(z)+\langle v, z\rangle} \\
& =(-1)^{\langle v, u\rangle} W_{f}(v) .
\end{aligned}
$$

Как видим, носитель спектра функции $f^{\prime}$ совпадает с носителем спектра функции $f$, только некоторым образом меняются знаки плюс и минус.

Для функции $f_{0}$ из рекурсивного класса будут выполнены следующие соотношения, справедливость которых доказывается непосредственной проверкой:

$$
\begin{gathered}
\operatorname{deg}\left(f_{0}\right)=2, \quad J_{T_{4}}\left(f_{0}\right)=\{(0,0,0,0),(1,1,1,1)\}, \\
L_{f_{0}}=\{(0,0,0,0),(1,1,0,0),(0,0,1,1),(1,1,1,1)\} .
\end{gathered}
$$

Лемма 4. Для функции $f_{k}, k=0,1, \ldots$, из рекурсивного класса,

$$
\operatorname{deg}\left(f_{k}\right)=2+k
$$

для любого $a=(u, v, w) \in L_{f_{k}}, u$ вес $\operatorname{wt}(a)$ четный.

Доказательство. Первое соотношения непосредственно вытекает из вида рекурсивного класса.

Доказательство второго соотношения проведем индукцией по $k$. Для $k=0$ все утверждения выполнены, это случай $f_{0}$, он разобран выше. Предположим, что соотношение выполняется для всех $k=0,1, \ldots, t-1$. Покажем, что оно выполнено и для $k=t$. 
Предположим противное. Пусть $a=(u, v, w) \in L_{f_{t}}$ и вес $\operatorname{wt}(a)=2 s+1$ нечетный. Поскольку $a \in L_{f_{t}}$, выполнено соотношение

$$
\begin{aligned}
& f_{t}(x+u, y+v, z+w)+f_{t}(x, y, z) \\
& =\left(z_{1}+z_{2}+w_{1}+w_{2}+1\right)\left[f_{t-1}(x+u)+\left\langle 1^{n_{t-1}}, y+v\right\rangle\right] \\
& \quad+\left(z_{1}+z_{2}+w_{1}+w_{2}\right)\left[f_{t-1}(y+v)+\left\langle 1^{n_{t-1}}, x+u\right\rangle\right]+z_{1}+w_{1} \\
& \quad+\left(z_{1}+z_{2}+1\right)\left[f_{t-1}(x)+\left\langle 1^{n_{t-1}}, y\right\rangle\right] \\
& \quad+\left(z_{1}+z_{2}\right)\left[f_{t-1}(y)+\left\langle 1^{n_{t-1}}, x\right\rangle\right]+z_{1}=\delta,
\end{aligned}
$$

где $\delta$ - константа. Преобразуем это соотношение:

$$
\begin{aligned}
& \left(z_{1}+z_{2}+w_{1}+w_{2}+1\right) f_{t-1}(x+u)+\left(z_{1}+z_{2}+1\right) f_{t-1}(x) \\
& \quad+\left(z_{1}+z_{2}+w_{1}+w_{2}\right)\left\langle 1^{n_{t-1}}, x+u\right\rangle+\left(z_{1}+z_{2}\right)\left\langle 1^{n_{t-1}}, x\right\rangle \\
& =\left(z_{1}+z_{2}+w_{1}+w_{2}\right) f_{t-1}(y+v)+\left(z_{1}+z_{2}\right) f_{t-1}(y) \\
& \quad+\left(z_{1}+z_{2}+w_{1}+w_{2}+1\right)\left\langle 1^{n_{t-1}}, y+v\right\rangle \\
& \quad+\left(z_{1}+z_{2}+1\right)\left\langle 1^{n_{t-1}}, y\right\rangle+w_{1}+\delta .
\end{aligned}
$$

Покажем, что действительно, если $\operatorname{wt}(w)$ нечетен, то выражение (10) выполнено быть не может. Из нечетности $\operatorname{wt}(w)$ следует, что $w_{1}+w_{2}+1=0$. При любой фиксации $z_{1}$ и $z_{2}$ в левой части выражения (10) останется многочлен степени, большей 2 , либо $f_{t-1}(x+u)$, либо $f_{t-1}(x)$. В правой части $x$ нет и из-за единственности полиномиального представления такого быть не может. Следовательно, wt $(w)$ четный. В этом случае выражение (10) принимает следующий вид:

$$
\begin{aligned}
\left(z_{1}+z_{2}+1\right)\left[f_{t-1}(x+u)+f_{t-1}(x)\right]+\left(z_{1}+z_{2}\right)\left\langle 1^{n_{t-1}}, u\right\rangle \\
=\left(z_{1}+z_{2}\right)\left[f_{t-1}(y+v)+f_{t-1}(y)\right]+\left(z_{1}+z_{2}+1\right)\left\langle 1^{n_{t-1}}, v\right\rangle+w_{1}+\delta .
\end{aligned}
$$

Фиксируя $z_{1}=z_{2}$ и используя предположение индукции о четности $w t(v)$, получаем, что $f_{t-1}(x+u)+f_{t-1}(x)$ есть константа, это значит, что $u \in L_{f_{t-1}}$. Аналогично для $z_{1} \neq z_{2}$ получаем, что $v \in L_{f_{t-1}}$. В итоге получаем, что вес $\operatorname{wt}(a)$ тоже четный. Лемма доказана.

Рассмотрим утверждение, описывающее строение группы инерции функции $f_{k+1}$.

Теорема 3. Пусть $f_{k}$ из введенного выше рекурсивного класса, тогда

$$
\begin{aligned}
& J_{T_{n_{k+1}}}\left(f_{k+1}\right)=J_{T_{n_{k}}}\left(f_{k}\right) \times J_{T_{n_{k}}}\left(f_{k}\right) \times\{(0,0)\} \\
& \cup\left(L_{f_{k}} \backslash J_{T_{n_{k}}}\left(f_{k}\right)\right) \times\left(L_{f_{k}} \backslash J_{T_{n_{k}}}\left(f_{k}\right)\right) \times\{(1,1)\} .
\end{aligned}
$$

Доказательство. Необходимо доказать включение в обе стороны. Докажем включение ว. Пусть

$$
a=(u, v, w) \in J_{T_{n_{k}}}\left(f_{k}\right) \times J_{T_{n_{k}}}\left(f_{k}\right) \times\{(0,0)\},
$$

тогда, используя четность веса $a$ из леммы 4 , получим цепочку равенств

$$
\begin{aligned}
f_{k+1}(x+u, y+v, z+w)= & \left(z_{1}+z_{2}+1\right)\left(f_{k}(x+u)+\left\langle 1^{n_{k}}, y+v\right\rangle\right) \\
& +\left(z_{1}+z_{2}\right)\left(f_{k}(y+v)+\left\langle 1^{n_{k}}, z+u\right\rangle\right)+z_{1} \\
= & \left(z_{1}+z_{2}+1\right)\left(f_{k}(x)+\left\langle 1^{n_{k}}, y\right\rangle+\left\langle 1^{n_{k}}, v\right\rangle\right) \\
& \quad+\left(z_{1}+z_{2}\right)\left(f_{k}(y)+\left\langle 1^{n_{k}}, z\right\rangle+\left\langle 1^{n_{k}}, u\right\rangle\right)+z_{1} \\
= & \left(z_{1}+z_{2}+1\right)\left(f_{k}(x)+\left\langle 1^{n_{k}}, y\right\rangle\right) \\
& \quad+\left(z_{1}+z_{2}\right)\left(f_{k}(y)+\left\langle 1^{n_{k}}, z\right\rangle\right)+z_{1} \\
= & f_{k+1}(x, y, z) .
\end{aligned}
$$


Аналогично разберем случай, когда

$$
a=(u, v, w) \in\left(L_{f_{k}} \backslash J_{T_{n_{k}}}\left(f_{k}\right)\right) \times\left(L_{f_{k}} \backslash J_{T_{n_{k}}}\left(f_{k}\right)\right) \times\{(1,1)\} .
$$

В этом случае

$$
\begin{aligned}
f_{k+1}(x+u, y+v, z+w)=( & \left.z_{1}+1+z_{2}+1+1\right)\left(f_{k}(x+u)+\left\langle 1^{n_{k}}, y+v\right\rangle\right) \\
& +\left(z_{1}+1+z_{2}+1\right)\left(f_{k}(y+v)+\left\langle 1^{n_{k}}, z+u\right\rangle\right) \\
& +z_{1}+1 \\
=( & \left.z_{1}+z_{2}+1\right)\left(f_{k}(x)+1+\left\langle 1^{n_{k}}, y\right\rangle+\left\langle 1^{n_{k}}, v\right\rangle\right) \\
& +\left(z_{1}+z_{2}\right)\left(f_{k}(y)+1+\left\langle 1^{n_{k}}, z\right\rangle+\left\langle 1^{n_{k}}, u\right\rangle\right) \\
& +z_{1}+1 \\
= & \left(z_{1}+z_{2}+1\right)\left(f_{k}(x)+\left\langle 1^{n_{k}}, y\right\rangle\right) \\
& +\left(z_{1}+z_{2}\right)\left(f_{k}(y)+\left\langle 1^{n_{k}}, z\right\rangle\right)+\left(z_{1}+z_{2}+1\right) \\
& +\left(z_{1}+z_{2}\right)+z_{1} \\
= & \left(z_{1}+z_{2}+1\right)\left(f_{k}(x)+\left\langle 1^{n_{k}}, y\right\rangle\right) \\
& +\left(z_{1}+z_{2}\right)\left(f_{k}(y)+\left\langle 1^{n_{k}}, x\right\rangle\right)+z_{1} \\
= & f_{k+1}(x, y, z) .
\end{aligned}
$$

Таким образом, в обоих случаях $a \in J_{T_{n_{k+1}}}\left(f_{k+1}\right)$.

Докажем включение $\subset$. Допустим, что $a=(u, v, w) \in J_{T_{n_{k+1}}}\left(f_{k+1}\right)$. Тогда справедливо равенство (10) с тем условием, что $\delta=0$. По лемме 4 , вес $w$ четный. Пусть $w=(0,0)$ и $z_{1}=z_{2}$, тогда из равенства (10) получим, что

$$
\left[f_{k}(x+u)+f_{k}(x)\right]=0 \Longrightarrow u \in J_{T_{n_{k}}}\left(f_{k}\right),
$$

а если $z_{1} \neq z_{2}$, то

$$
\left[f_{k}(y+v)+f_{k}(y)\right]=0 \Longrightarrow v \in J_{T_{n_{k}}}\left(f_{k}\right) .
$$

Пусть теперь $w=(1,1)$. Рассматривая случаи $z_{1}=z_{2}$ и $z_{1} \neq z_{2}$, получаем, что $u \in L_{f_{k}} \backslash J_{T_{n_{k}}}\left(f_{k}\right), v \in L_{f_{k}} \backslash J_{T_{n_{k}}}\left(f_{k}\right)$. Здесь мы использовали то, что веса $\operatorname{wt}(u)$, $\operatorname{wt}(v)$ четные, и поэтому

$$
\left\langle 1^{n_{k-1}}, v\right\rangle=\left\langle 1^{n_{k-1}}, u\right\rangle=0 .
$$

Таким образом, включение $\subset$ выполняется. Теорема доказана.

Лемма 5. Пусть $f_{k}$ из определенного выше рекурсивного класса, тогда

$$
\left|\left\{f_{k}\right\}_{T_{n_{k}}}\right|=2^{4 \cdot 2^{k}-1} .
$$

Доказательство. Воспользуемся следующими соотношениями, доказанными в [2]:

$$
\begin{aligned}
& \left|L_{f_{k}}\right|=2\left|J_{T_{n_{k}}}\left(f_{k}\right)\right|, \\
& \left|f_{T_{n}}\right|=\frac{\left|T_{n}\right|}{\left|J_{T_{n}}(f)\right|} .
\end{aligned}
$$

Поскольку

$$
\left|L_{f_{k}} \backslash J_{T_{n_{k}}}\left(f_{k}\right)\right|=\left|J_{T_{n_{k}}}\left(f_{k}\right)\right|,
$$


из теоремы 2 вытекает справедливость цепочки равенств

$$
\begin{aligned}
\left|J\left(f_{k}\right)\right| & =2\left|J\left(f_{k-1}\right)\right|^{2}=2\left(2\left|J\left(f_{k-2}\right)\right|^{2}\right)^{2} \\
& =2^{1+2+4+\ldots+2^{k}}=2^{2^{k+1}-1} .
\end{aligned}
$$

Зная, что

$$
\begin{aligned}
\left|T_{n_{k}}\right| & =2^{n_{k}}, \\
n_{k} & =6 \cdot 2^{k}-2,
\end{aligned}
$$

получаем, что

$$
\begin{aligned}
\left|\left\{f_{k}\right\}_{T_{n_{k}}}\right| & =\frac{\left|T_{n_{k}}\right|}{\left|J_{T_{n_{k}}}\left(f_{k}\right)\right|}=\frac{2^{n_{k}}}{2^{2^{k+1}+1}} \\
& =2^{6 \cdot 2^{k}-2-22^{k}+1}=2^{4 \cdot 2^{k}-1} .
\end{aligned}
$$

Лемма доказана.

Теорема 4. Множество платовидных функиий с носителем спектра, описываемым матрицей $A_{k}$, совпадает с классом эквивалентности функции $f_{k}$ относительно группьл сдвигов, то есть $M_{k}=\left\{f_{k}\right\}_{T_{n_{k}}}$.

Доказательство. Из леммы 3 видно, что $\left\{f_{k}\right\}_{T_{n_{k}}} \subset M_{k}$. Но поскольку мощности этих множеств совпадают, получаем, что они равны. Это доказывает теорему.

Таким образом, класс $M_{k}$ платовидных булевых функций, задаваемый матрицей носителя спектра $A_{k}$, представляет собой класс эквивалентности функции $f_{k}$ относительно группы сдвигов $T_{n_{k}}$.

\section{Список литературы}

1. Таранников Ю. В., О значениях аффинного ранга носителя спектра платовидной функции. Дискретная математика (2006) 18, №3, 120-147.

2. Логачев О. А., Сальников А. А., Ященко В. В., Булевы функции в теории кодирования и криптографии. МЦНМО, Москва, 2004.

3. Таранников Ю. В., О корреляционно-иммунных и устойчивых булевых функциях. Математические вопросы кибернетики (2002) 11, 91-148.

Статья поступила 6.09.2010. 